\title{
Path State-Based Update of PCE Traffic Engineering Database in Wavelength Switched Optical Networks
}

\author{
Alessio Giorgetti, Filippo Cugini, Nicola Sambo, Francesco Paolucci, \\ Nicola Andriolli, and Piero Castoldi, Member, IEEE
}

\begin{abstract}
This letter proposes to use the path state approach for updating the Traffic Engineering Database (TED) of the Path Computation Element (PCE) in GMPLS controlled Wavelength Switched Optical Networks (WSONs). The proposed solution reduces both the lightpath blocking probability and the control plane load with respect to conventional updating schemes based on the link state approach.
\end{abstract}

Index Terms-PCE, OSPF-TE, WSON.

\section{INTRODUCTION}

$\mathbf{T}$ HE Path Computation Element (PCE) has been recently introduced in dynamic GMPLS controlled Wavelength Switched Optical Networks (WSONs) for overcoming the limitations of fully distributed routing and wavelength assignment (RWA) [1]. In particular, the PCE provides effective network resource allocation, and simplifies the implementation of network nodes, which may avoid complex routing modules.

The PCE utilizes a Traffic Engineering Database (TED) to perform path computation and, possibly, wavelength assignment decisions. It is important that this TED is accurate and updated when the PCE performs a path computation. In particular, the use of detailed wavelength availability information is fundamental in WSONs to obtain adequate performance [2], [3].

Traditionally, the PCE TED is updated by a link state routing protocol, i.e., the Open Shortest Path First routing protocol with Traffic Engineering extensions (OSPF-TE). However, current OSPF-TE implementations do not support the advertisement of detailed wavelength availability information. Moreover, further information may be required by the PCE, such as switching node architectural asymmetries and wavelength connectivity constraints, whose flooding through OSPFTE can overload the control plane and cause convergence issues [4].

Alternative approaches for updating the PCE TED are therefore necessary. In particular, the work in [4] proposes a link state approach, where each network node directly communicates to the PCE the information about the state of locally connected links.

This letter proposes two schemes using the path state approach for PCE TED updating, in which the network nodes

Manuscript received February 18, 2010. The associate editor coordinating the review of this letter and approving it for publication was N. Ghani.

This work was carried out with the support of the BONE-project (Building the Future Optical Network in Europe), a Network of Excellence funded by the European Commission through the 7th ICT-Framework Programme.

A. Giorgetti, N. Sambo, F. Paolucci, N. Andriolli, and P. Castoldi are with Scuola Superiore Sant'Anna, Pisa, Italy (e-mail: alessio.giorgetti@sssup.it).

F. Cugini is with CNIT, Pisa, Italy.

Digital Object Identifier 10.1109/LCOMM.2010.06.100291 directly communicate the information about established and released lightpaths to the PCE. This solution is able to provide an accurate and timely updated TED at the PCE, guaranteeing low blocking and strongly reducing the control plane load with respect to link state approaches.

\section{PCE-Based Lightpath Setup}

In PCE based WSONs, upon lightpath request, the source node exploits the PCE communication Protocol (PCEP) for submitting path computation requests to the PCE (i.e., using a PCEP PCReq message) [1]. In all the scenarios considered in this letter, the PCE maintains the TED with detailed wavelength availability information, i.e., the status (reserved or available) of each wavelength on every link. Therefore, besides path computation, the PCE also selects the wavelength to be used by the lightpath. Both the computed path and the selected wavelength are communicated to the source node by using a PCEP PCRep message. Conversely, if the PCE fails in computing a path with available resources, it replies with an error message and the lightpath request is refused (i.e., routing blocking).

Upon reception of the PCRep message, the source node triggers an instance of the Resource Reservation Protocol with Traffic Engineering extension (RSVP-TE) along the computed path, to actually reserve resources [5]. RSVP-TE is based on the utilization of Path and Resv messages, sent in the forward and backward directions, respectively. In the considered scenarios, the Path message includes the Explicit Route, the Suggested Label, and the Label Set standard objects [5]. The Explicit Route and the Suggested Label objects respectively contain the path and the wavelength selected by the PCE. The Label Set object is created at the source node and updated along the path so that, when the destination node is reached, it contains the wavelengths that are available on the end-to-end path. Upon reception of the Path message the destination node performs the wavelength assignment. In particular, the wavelength indicated in the Suggested Label object is selected if it is included in the Label Set. Otherwise, another wavelength contained in the Label Set is selected, according to a specific wavelength assignment strategy. After wavelength assignment, the destination node sends back a Resv message to effectively reserve the selected wavelength on each link of the path. Once the Resv message reaches the source, the lightpath is established and data transmission can take place.

Using the described procedure, the RSVP-TE instance may be blocked during both forward and backward signaling phases [6]. Blocking during the forward phase (i.e., forward 


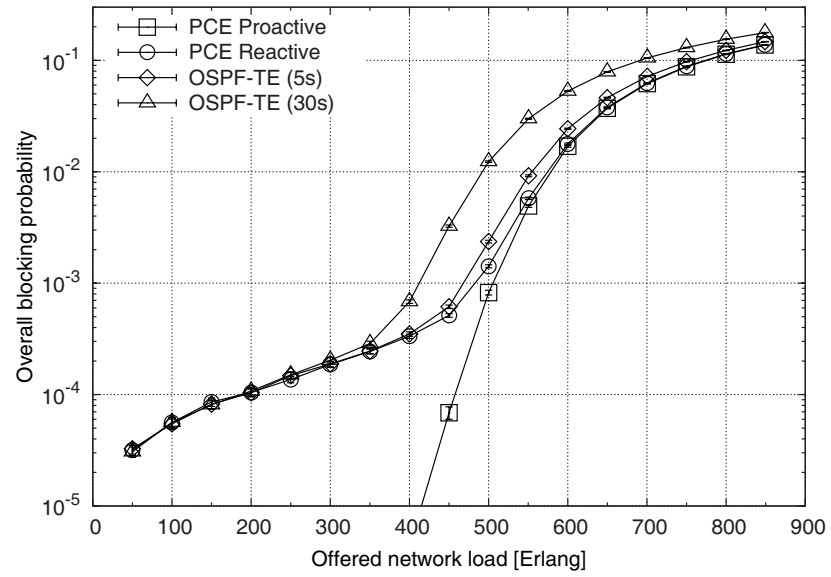

Fig. 1. Overall blocking probability.

blocking) is due to wavelength unavailability on the path. It happens when the updated Label Set results to be empty in an intermediate node or at destination. Blocking during the backward signaling (i.e., backward blocking) is due to wavelength contentions. Contentions are caused by the concurrent attempt of two or more RSVP-TE instances to reserve the same wavelength on the same link. Indeed, under dynamic traffic conditions, the list of available wavelengths contained in the Label Set object of the Path message may be already outdated when the destination node is reached.

\section{PCE TED UPDATE}

This letter proposes two path state based schemes (i.e., the PCE Reactive and the PCE Proactive), in which a direct communication between network nodes and the PCE is used to update the PCE TED with information regarding whole paths. The proposed schemes are compared with a link state based scheme in which the PCE updates its TED by sniffing the Link State Advertisements (LSAs) flooded by OSPF-TE (i.e., the OSPF-TE based).

- PCE Reactive: Every time a lightpath is established (released) the source node communicates to the PCE the reserved (freed) wavelengths along the path. In case of errors during the RSVP-TE signaling no communication between the source node and the PCE is required.

- PCE Proactive: Every time the PCE successfully performs a path computation, it updates its TED assuming the successful lightpath establishment along the computed path on the suggested wavelength. The source notifies the PCE, which consequently updates its TED, in three different cases: 1) if an error occurs during the RSVP-TE signaling; 2) if the lightpath is established using a different wavelength; 3) when the lightpath is released.

- OSPF-TE based: With this scheme LSAs are generated by network nodes when a wavelength status change occurs on a link. However, once an LSA has been generated for a given link, any change detected on such link before a timeout is elapsed is not advertised [7].

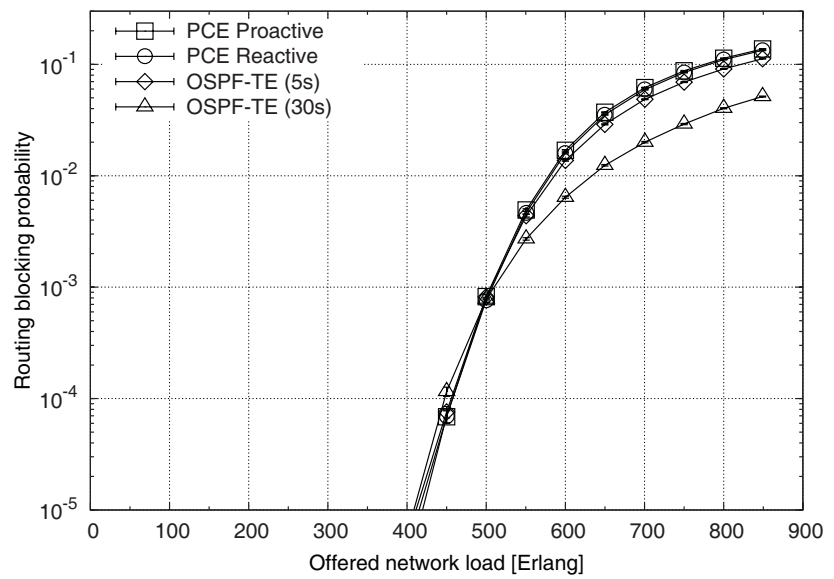

Fig. 2. Routing blocking probability.

\section{Simulation Study}

Simulations are performed with a custom-built C++ eventdriven simulator. A Pan-European network topology is considered with 27 nodes, 55 bidirectional WDM links and 32 wavelengths per link along each direction. Nodes do not support wavelength conversion. Lightpath requests are generated according to a Poisson process and uniformly distributed among all node pairs. Both, inter-arrival and holding times are exponentially distributed. The average holding time is fixed to 180 seconds. Two values of the LSA update timeout are considered respecting the OSPF-TE standard [7]: 5 and 30 seconds, thus resulting in two versions of the OSPF-TE based scheme: $O S P F-T E(5 s)$ and $O S P F-T E(30 s)$. All the simulation points are depicted with the reached confidence interval at 95\% confidence level.

In all the considered scenarios, the PCE uses the information stored in the TED for performing the path computation and the selection of the suggested wavelength. In particular, for a lightpath request between the node pair $(s, d)$, the PCE selects the path within a set $P_{s, d}$ of candidate paths. The path in $P_{s, d}$ that can accommodate the largest number of wavelength-continuous lightpaths is selected [3]. Possible ties are randomly broken. The set $P_{s, d}$ includes all the paths whose hop length is within one hop from the shortest path. In addition, the PCE randomly selects the suggested wavelength among all the wavelengths that are seen as available on the whole path. If, during the signaling instance, the suggested wavelength is detected as not available, the destination node randomly selects a wavelength in the received Label Set object.

Fig. 1 depicts the overall blocking probability versus the offered network load. The figure shows that the PCE Proactive update achieves the best performance guaranteeing almost null blocking up to 400 Erlang. The other three schemes provide not negligible blocking also under very light traffic conditions. With this load the blocking is mainly due to wavelength contentions (see Fig. 3). At higher loads, with faster arrivals, the OSPF-TE (30s) scheme provides the highest blocking. Indeed, with such a dynamic traffic, a 30 seconds timeout is not adequate to guarantee a timely TED. On the contrary, both the proposed schemes guarantee an updated TED, resulting in better blocking performance with all traffic loads. 


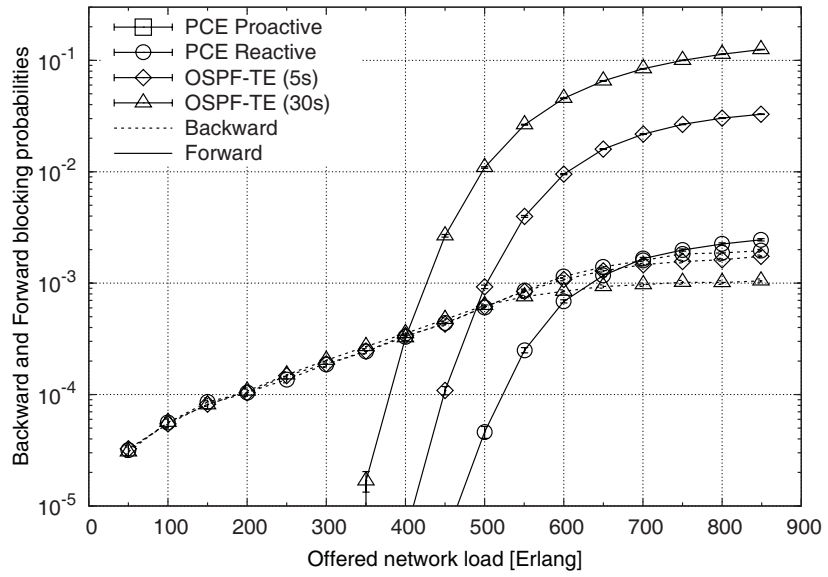

Fig. 3. Backward and forward blocking probabilities.

Fig. 2 depicts the routing blocking probability versus the offered network load. Routing blocking appears at high loads, indeed it occurs when the PCE is not able to find a path with available resources. The OSPF-TE (30s) scheme provides the best routing blocking mainly due to the lower accepted load, testified by the highest overall blocking (see Fig. 1).

Fig. 3 depicts the backward and the forward blocking probabilities versus the offered network load. First, the plot shows that backward blocking is the dominant source of blocking at low and medium loads. Moreover, it shows that the two OSPF-TE based schemes and the PCE Reactive scheme provide very similar backward blocking, which increases with increasing load, when arrivals are faster. On the contrary, the PCE Proactive scheme does not experience any backward blocking. This is because the PCE Proactive considers network resources to be busy immediately after the path computation so that successive lightpath requests are routed avoiding the resources allocated to previously computed paths. Fig. 3 also shows a very different forward blocking for the four schemes. Forward blocking occurs when the PCE considers available network resources that are currently reserved. This happens in the OSPF-TE schemes for delayed LSAs, and in the PCE Reactive due to ongoing signaling instances. Indeed, the OSPFTE (30s), scheme provides the highest blocking followed by the OSPF-TE (5s) scheme, and by the PCE Reactive scheme. Finally, the PCE Proactive scheme achieves also null forward blocking. Indeed, with the used update process, the TED is updated before actual resource reservation, so that a busy resource is never considered as available.

Fig. 4 depicts the control plane load expressed as the average bitrate generated by processed control messages (i.e., RSVP-TE, OSPF-TE, PCEP) at each node, versus the offered network load. Fig. 4 shows that the OSPF-TE based schemes generate an higher control plane load with respect to the proposed path state based schemes. Indeed, the proposed schemes aggregate the notification of wavelength status changes on a per path base, moreover each change is not advertised throughout the all network but directly communicated to the PCE.

Finally, the PCE Proactive scheme slightly reduces the control plane load generated by the PCE Reactive scheme. Indeed, the former triggers a notification to the PCE when there is

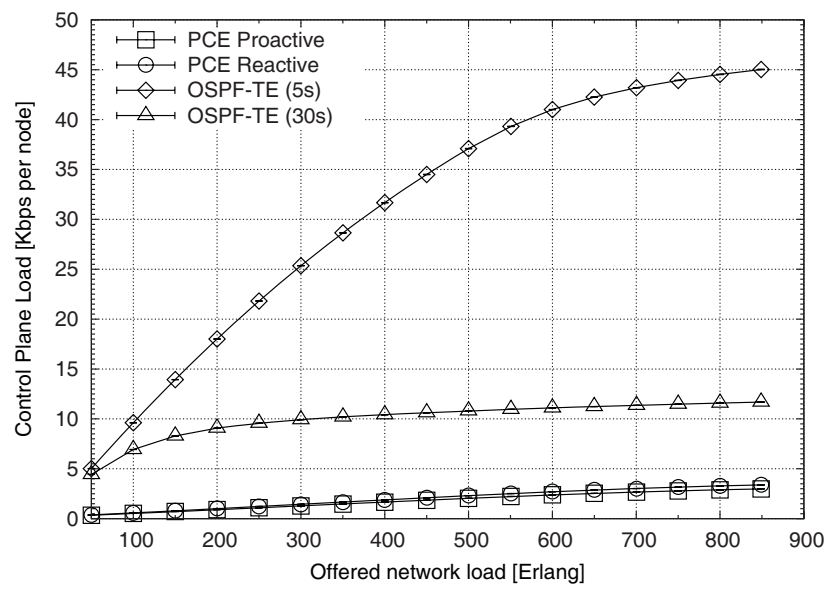

Fig. 4. Average control plane bitrate per node.

an error during the RSVP-TE signaling or the lightpath is established using a wavelength different from the wavelength suggested by the PCE. The latter requires a communication to the PCE each time a lightpath is correctly established. Since the correct lightpath establishment is the most frequent case, the PCE Proactive scheme requires a reduced amount of notification messages to the PCE and therefore achieves the lightest control plane load.

\section{CONCLUSION}

This letter proposed the use of the path state approach for updating the PCE TED using notification messages (e.g., PCEP Notify or RSVP-TE Notify) generated upon lightpath establishment and release.

Simulation results show that the proposed approach strongly reduces the control plane load, and significantly reduces the lightpath blocking probability. In particular, the PCE Proactive update scheme guarantees the best performance by avoiding any blocking during the signaling phase, thus jointly achieving the best blocking and the lowest control plane load.

\section{REFERENCES}

[1] V. Lopez, B. Huiszoon, J. Fernandez-Palacios, O. Gonzalez de Dios, and J. Aracil, "Path computation element in telecom networks: recent developments and standardization activities," in Proc. ONDM 2010, Jan. 2010.

[2] G. Bernstein and Y. Lee, "Extending GMPLS/PCE for use in wavelength switched optical networks," in Tech. Dig. OFC 2008, Mar. 2008.

[3] A. Giorgetti, N. Sambo, I. Cerutti, and P. Castoldi, "Impact of link-state advertisement in GMPLS-based wavelength-routed networks," in Tech. Dig. OFC 2008, Mar. 2008.

[4] Y. Lee G. and Bernstein, "Alternative approaches to traffic engineering database creation and maintenance for path computation elements," IETF draft-lee-pce-ted-alternatives-02.txt, May 2009.

[5] L. Berger, "Generalized multi-protocol label switching (GMPLS) signaling resource reservation protocol-traffic engineering (RSVP-TE) extensions," IETF RFC 3473, Jan. 2003.

[6] A. Giorgetti, N. Sambo, I. Cerutti, N. Andriolli, and P. Castoldi, "Label preference schemes for lightpath provisioning and restoration in distributed GMPLS networks," J. Lightw. Technol., vol. 27, no. 6, pp. 688697, Mar. 2009.

[7] D. Katz, K. Kompella, and D. Yeung, "Traffic engineering (TE) extensions to OSPF version 2," IETF RFC 3630, Sep. 2003. 